\title{
Enriching Curricula with Mobile Solutions
}

\author{
Dinu MIHAIL-VĂDUVA \\ Bucharest University of Economic Studies \\ dinu.mihailvaduva@stud.ase.ro
}

The most enduring appeal of a mobile learning application is to enrich a curriculum regardless a specific domain of study. Mobile technology is a very versatile industry with quick connection to any untethered requirements coming from the realm of teaching activities. This article is dedicated to transfer a general understanding of concepts linked to a statistics course wrapped with a good knowledge and practice about programming languages used in mobile devices. For this objective we are starting a quest for finding relevant references through scientific articles regarding the main practically intertwined features between statistics, programming languages and teaching activities. We are trying to show that developing an Android mobile application is a practical solution that, in a simultaneously way, helps teachers to do a better teaching and evaluating activities and, besides that, speaking about students, we explain that reading, understanding and solving specific issues is a key target. We find that scientific community involved into m-learning subject is convinced that a higher exam scores is obtained, against traditional methods of doing lessons, when an evaluation regarding learning activity occurs. This paper contains the salient facts about creating an Android mobile application considering basic concepts taken from econometrics.

Keywords: Mobile applications, Mobile learning, Android, Quality metrics, Econometrics

1 Introduction

A study conducted by Zahra [1] reveals the relationship between teaching lessons with large utilization of mobile devices and students' motivation for doing a better learning activity. Their results show that entanglement between students' ideas were in a positively correlation with the situations where technology of mobile devices was used. The fact that a mobile device is a cheaper acquisition than a desktop or laptop computer and offers connectivity with wireless network anytime and anywhere is certainly an important cause to this trend revealed by many scientific research papers. This mobile attitude shift is a source for considering a new age of learning on-the-go or just-in-time learning by Zoraini [2]. A possible explanation came from Barbaux [3] who observed that young generation born simultaneously with new digital technology. New educational applications were developed for mobile devices because these products bring certain improvements about reading and writing students' skills, more attention when solving problems, better memory and a positive attitude about readiness to trust in his/her own powers.

Between active learning and working actively could be a diversity of approaches but the point of intersection is considered to be that students [4] are engaged to complete certain requirements. From study written by [5] conducted us the conclusion that generating and then analyzing statistical data is the main source to improve understanding of the statistical concepts. If we are using regression analysis build with a mobile device then we create a dynamic lesson that could increase the focus of a learner. The findings described here could be used to help readers designing a similar mobile project to ensure a better content for statistical educational purpose.

The paper is structured, as follows. The first section is a general introduction to this domain and explains the objective of this paper. The second section is a review of scientific literature that describes connections between other research efforts linked to our specific objective, especially those regarding m-learning and econometrics. The third section presents the methodology that we used for this research and shows an Android application using Java language. In this 
section we show the general architecture of our application and theoretical concepts about exploratory analysis with econometrics. The fourth chapter explains a personal study correlated with a software model to help solving and understanding statistical characteristics of a variable and linear regression. In the first paragraph of this section, appointed by 4.2, we introduce basic mathematics formulas used in our application. In the second paragraph, appointed by 4.2 , we show with specific details how we design our mobile application concerning architecture, logical and physical implementation and a general view about using tools for developing apps inside Android Studio. In the fifth chapter we explain why a cumulative approach for both academic branch studied at our university, econometrics and language programming for mobile devices, could be a better solution to develop practical lessons. In the sixth chapter we deploy our conclusions and details about other directions that we intend to follow regarding our master project.

\section{Literature review}

For an experience about how to use and produce useful results from the study of econometrics using m-learning we mention general considerations described in the literature [6] . Working actively is a must for any student and requires skills to complete an oral and written presentation, working in a team and self-evaluation. There are specific activities like answering and elaborating questions, solving problems, searching through scientific articles and attending to relevant meetings where other colleagues or teachers are involved in the similar projects. Other article, written by [7], identifies a new educational framework where the teacher is not a main source and the student is not a destination when we speak about knowledge. Here we find a description for a mobile software tool named FTOOL (Two Dimension Frame Analysis Tool) with practical implementation for an engineering course named Structural Analysis I of the 3h year in the Faculty of Engineering of the University of Porto. This study shows the important role of the mobile multimedia interactive resources related to a proper response against the failure of student's performance. For an economic goal about regression calculus, a research conducted by Tilca [8] shows the way concerning how to evaluate the rank of a business using a developed mobile Android application. This paper [8] explains the process of developing an Android software application with an exception of the publishing step. An interesting research finished by [9] reveals how to create an educational platform, what could be loaded even by a mobile device, to optimize the performance of a life-long learning activity. From the presented examples is easy to see the cross-disciplinary characteristics of doing a research that involves teaching and evaluation related with any subject of knowledge. There are three factors influencing m-learning, according with [10], which could be synthesized remembering the features of the mobile devices, user's preferences and educational advantage. About any mobile device is well known that usability, functional and technical aspects refer to instant information at different locations, quickly search, no restrictions regarding time and position and gaining learning documents from various places. Learning mathematics with mobile learning opens new opportunities to develop an increasing motivation of studying econometrics. Using ANOVA [1] the level of teachers' education and experience was correlated with a positive effect about students' participation in Mathematics. The major results of research [1] explain that teachers of Mathematics are involved in deploying mobile devices for learning activities in Mathematics. Evaluation on mobile learning was examined [2] and learners $(42.38 \%)$ have the opinion that an activity of learning with a mobile device will produce better results regarding their time management. In percentage terms, a value of $45.06 \%$, among learners, agreed that $\mathrm{m}$ learning is meaning a good reason to stay focused much longer due to a minimum faceto-face interaction with their teachers and 
colleagues. From a pedagogical point of a teacher's view [3] there are four foundation concepts consolidated around context, conversation, collaboration and confluence. The context is meaning that information has to be displayed in small units named 'chunks' to accept a smaller display, specific processing power of mobile phones and students' shorter times for learning. A conversation could be a dialogue between students teaching personnel inside a space with multiple attributes for sharing understandings, testing hypotheses and making productive a virtual environment. Cooperation is a sum of activities generated by putting together many collaborative tasks to create a community, a new identity to achieve common goals. A confluence of different inputs like web sites, emails, TV channel, RSS feeds provide the chance to determine a higher speed of information and more random access. This heterogeneous environment maintains a vision of 'constellated' resources where tasks completed by students would supplementary added to each other the most relevant options. Maintaining the students focused is a goal [11] mentioned as a primary target in BUES for learning analytics because feedback make a quality difference when we speak about educational process. Using regression analysis is mentioned by [12] when a research team studied how children's math achievement in different classes could be related with gender and race. A phenomenon frequently discussed is about adults' participation rates correlated with non-formal educational activities [13] and the conclusion is that public sector attracts learners because more qualitative services, socialization and making new friends. On the other hand, the private sector attracts candidates willing to learn because those people expressed a genuine fear not to lose a job. Another example of using econometric analysis upon mobile internet statistics was made by [14] regarding two basic trends such as Mobilefriendly algorithm update and Mobile-first index. They concluded that the market maturity have a major controlling influence on how the Internet is used based on the data published in November 2016 by StatCounter. According to this independent Web analytics, mobile platforms [14] have a rapid growth considering traffic generated by monitored web pages.

A research about designing learning environments with the help of mobile devices has worthwhile to be mentioned due to a great interest allocated to build a technology roadmap that assures effectiveness, productiveness [15] and those aspects will generate a high quality of education. When we consider the domain of language learning it is a major contribution from a research team [16] with their noticeable paper work in this field. They remark that a mobile device ensures a great impact for learners because the target language has a continuous relationship with them. Any learner that use a mobile device as a tool could be seen in a dual perspective by reason of joining two wellknown statement such as learning from mobile and learning with mobile. This point of view was analyzed by [17] in a research paper whose magnifying effort build a delimitation between using a mobile device to deliver learning materials and, a second situation, when a mobile device assures a higher level of thinking skills.

\section{Methodology}

This study was realized to establish how to create a mobile solution (targeting Android platform) to emulate practical lessons about econometrics, like describing quantitative variables and making prediction using regression analysis. When we speak about quantitative variables it is important to explain those methods that measure location and variability of them, such as mean, sample variance, standard deviation and the coefficient of variation if we want to be able to perform exploratory analysis. This practical approach is required when we desire to compare different groups of values previously allocated to a series of instances regarding to the same procedure or event. For regression analysis we consider the least squared method to calculate simple linear regression. With 
simple linear regression we foresee scores on one variable, named criterion variable and referred with Y, using another variable, named predictor variable and referred with $X$. This procedure creates a straight line between $\mathrm{Y}$ and $\mathrm{X}$.

For this research, we combine a practical approach with a theoretical one. The students have to develop programming skills for Android platform using Java programming language. It is created around the Linux kernel and applications are written in Java and the packaged together with any data a resource files. There is a different virtual machine named Dalvik which is registered based instead using a stack conception as standard Java VM. For Dalvik method we know that every time an android app is running, JIT (Just in time compilation) process is launched and the code required is going to be translated to the machine code. There is a new approach named ART desired to be a replacement to run time called Dalvik. This new method ART was introduced experimentally in the 4.4 release KitKat. The main difference between Dalvik and ART starts from the fact that ART method implies a supplementary compilation of the Dalvik code into a system dependent binary therefore will be no time needed for JIT compilation allocated every time when an app is running. The main component of an Android app is based on a single screen with a specific interface called Activity. Every application could have several activities, a single thread design and functions events oriented. The platform of Android was well equipped with a modern IDE ready to execute completion, analysis and refactoring of code. Any package application could be build, test and run using a virtual device manager that emulates common Android devices. The file format for any Android package designed to be used onto Google's Android OS is named apk. The application is written with the help of Java programming language and debugging is completed by android Debug Monitor. For those who prefer other alternatives to Java language and Google's tools there are different choices remembered here: Appcelerator with JavaScript, Cordova for
HTML5 or MonoGame and Xamarin used with C\#. A complete list to these alternatives could be found reading Fowler [18].

\section{Case Study}

\subsection{Application description}

For our project we create a software model to support solving and understanding statistical characteristics of a variable and linear regression. The last one is used to define a statistical relationship between a variable $\mathrm{Y}$ and $X$ which could be emulated using the equation $\mathrm{Y}_{\mathrm{i}}=\mathrm{a}+\mathrm{b} * \mathrm{X}_{\mathrm{i}}$. For a quantitative variable the average is a typical value of a sample population and could be calculated with well-known formula for the arithmetical mean. The sample variance is represented by the sum of all squared differences, between the value of a variable at one moment of time and the arithmetical mean, divided by an amount of one subtracted from the size of the population. This statistical characteristic is the most common way to describe the variability of a variable. The main unfavorable quality of the sample variance is the fact that it uses squared values of the variable. For that reason we use the standard deviation which is calculated using the square root of the sample variance. The formula for the sample variance is shown in (1) and the formula for the standard deviation is presented in (2).

$$
\begin{aligned}
S^{2} & =\frac{\sum_{i=1}^{n}\left(x_{i}-\bar{x}\right)^{2}}{n-1} \\
S & =\sqrt{S^{2}}=\sqrt{\frac{\sum_{i=1}^{n}\left(x_{i}-\bar{x}\right)^{2}}{n-1}}
\end{aligned}
$$

$x_{i}$ are the values of the variable and $n$ is the size of the population. Another unfavorable quality of using the sample variance is the fact that the variability cannot be translated between different units reported for the variable. In this case we use the coefficient of the variation which is the ratio of the standard deviation to the arithmetical mean of the population. On the other hand, the regression analysis is a method frequently used in statistics where the objective is to correlate values of an independent variable, named here 
$\mathrm{X}$, with a dependent variable $\mathrm{Y}$, trying to extract the average change of the $Y$ for one unit change in $\mathrm{X}$. The type of link between $\mathrm{Y}$ and $X$ is a statistical relationship not a functional one. The statistical relationship between $\mathrm{Y}$ and $\mathrm{X}$ is not known. For a functional link the relationship between $\mathrm{Y}$ and $\mathrm{X}$ is generated using a known formula. The general equation accepted, for a statistical link, is shown in the following formula that can be written: $Y_{i}=a+b * X_{i}$. This link is considered simple because we have only two variables. For an equation $\mathrm{y}=\mathrm{f}\left(\mathrm{x}_{1}, \mathrm{x}_{2} \ldots \mathrm{x}_{\mathrm{i}}\right)$ we say that the link is multiple. When difference for two consecutive values of $\mathrm{Y}$ and $\mathrm{X}$ have the same sign we say that the link is direct or positively correlated between $\mathrm{X}$ and $\mathrm{Y}$, otherwise we name it an inverse determination or negatively correlated. The formula is used when the link is linear but we could also find a situation where there is no linear correlation. It is possible to insert and to modify the properties for any object selected ensuring that the main purpose of the software application is achieved. The objective treated in our paper is linked to regression analysis. Our software task is to find the coefficient named $a$ and $b$ from this equation. The common notation for the above equation is:

- $\mathrm{Y}_{\mathrm{i}}$ refers to the outcome or dependent variable $\mathrm{Y}$

- $\mathrm{X}_{\mathrm{i}}$ refers to the predictor or independent variable $\mathrm{X}$

We know that such a link could be solved using the system presented in (3) where the coefficient named $n$ refers to all pairs of values $\left(Y_{i}, X_{i}\right)$.

$$
\left\{\begin{array}{c}
n a+b \sum x_{i}=\sum y_{i} \\
a \sum x_{i}+b \sum x_{i}^{2}=\sum x_{i} y_{i}
\end{array}\right.
$$

The system of equations used to determine the coefficients named $a$ and $b$. To test the strength between variable $\mathrm{Y}$ and $\mathrm{X}$ we choose to calculate the correlation coefficient $\mathrm{R}$, named Pearson using the formula shown in (4).

$$
R=\frac{n \sum x_{i} y_{i}-\sum x_{i} \sum y_{i}}{\sqrt{\left(n \sum x_{i}^{2}-\left(\sum x_{i}\right)^{2}\right)\left(n \sum y_{i}^{2}-\left(\sum y_{i}\right)^{2}\right)}}
$$

How does the Pearson's Correlation Coefficient (R) explain the statistical relationship between $\mathrm{Y}$ and $\mathrm{X}$ ? If this correlation coefficient is closer to 1 then we have a stronger positive linear relationship between variables $\mathrm{Y}$ and $\mathrm{X}$. If the correlation coefficient is closer to 0 then we have a weaker linear relationship.

\subsection{Application implementation}

This paper explains how to develop an Android application with Android Studio for a generic econometric purpose considering that interpretation of results is the most important objective. The development process starts with an idea and its requirements. Prototyping a user interface is meaning that we desire to show how the user would work. Developing an app begins with a layout which contains string resources and menu items written using the Extensible Markup Language (XML). Every activity must be declared in the manifest file. At the end we will choose a build configuration and assemble the final APK (package file). The basic architecture of the process of development using Android Studio is presented in Figure 1.

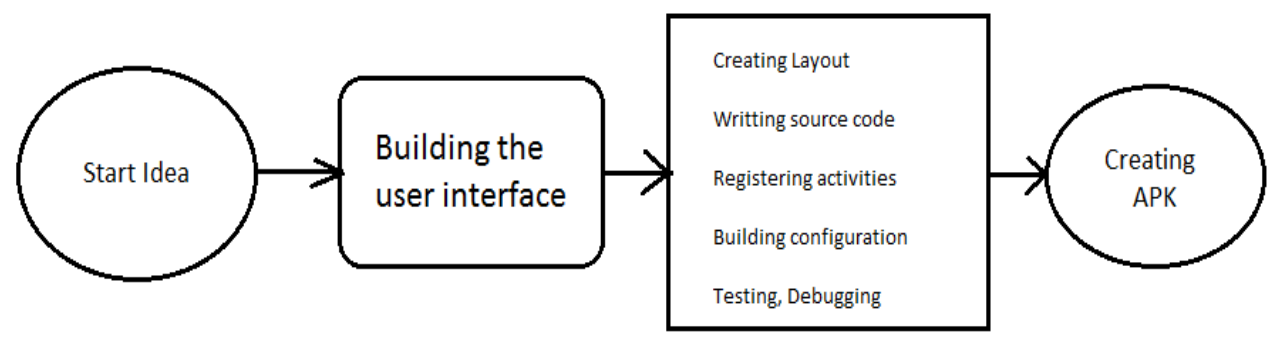

Fig. 1. The process of development for an application using Android Studio 
Every process of development begins with a thought or a plan for a possible software project and could be assimilated with a picture in the mind. This stage involves market and science research. The starting idea progresses and a new state appears in which the main focus is located upon building the user interface. Creating the layout is meaning that we place UI elements and uses the Extensible Markup Language (XML) to assign string resources required by every item stored into Android project. Writing the source code is completed using Java language by using testing and debugging tools. Declaring every activity in the manifest file is compulsory. Creating a default or a custom building configuration should be a choice correlated with the project's version. The final APK could be now assembled and this is the final stage when the product is transferred to the end-user.

The project structure is organized using groups according to preset Android view established by the Android Studio environment. These groups are presented in Figure 2.

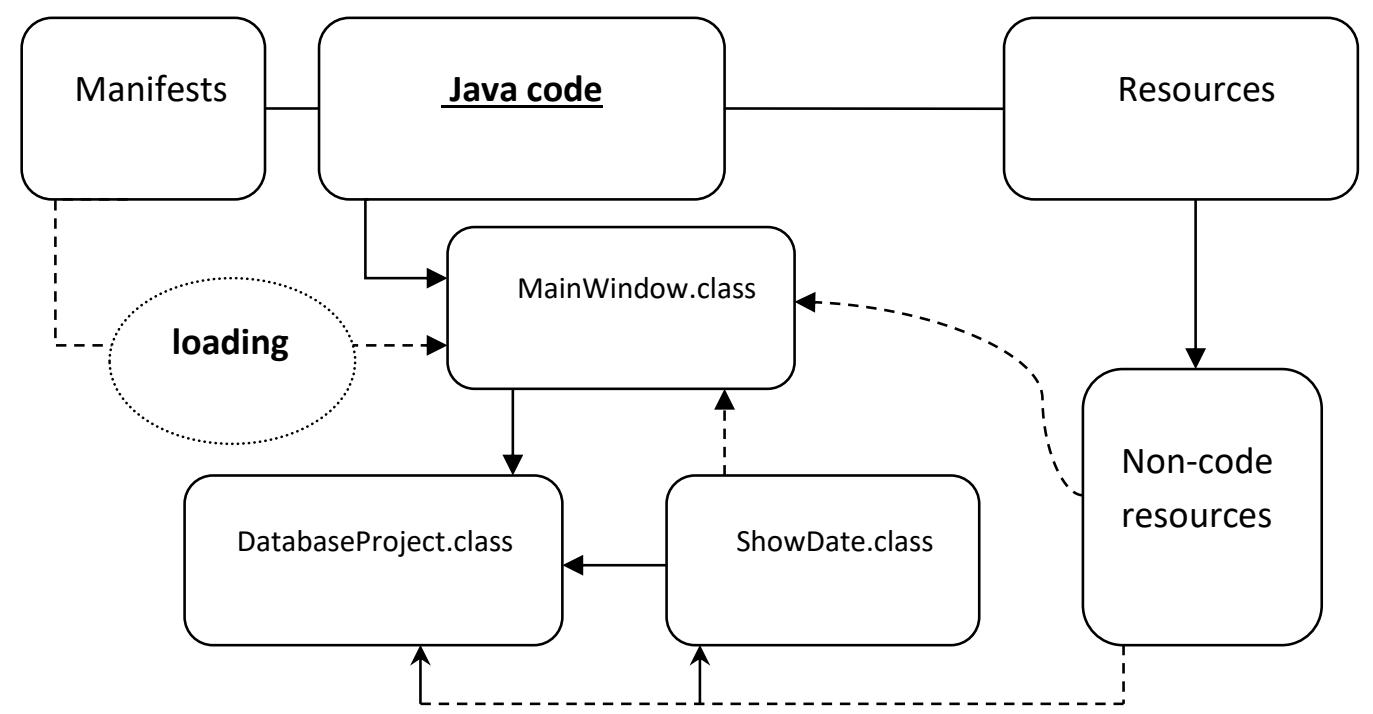

Fig. 2. The application components

These groups help the user to navigate more easily between source files of the project. The first group is called manifests and contains the AndroidManifest.xml file. This file offers a bridge between the software application and Android operating system. It contains information about Android version, access permissions and all components included in the application. The second group operates with the Java source code files grouped by package names and its name is java. The main activity declared by AndroidManifest.xml file is the Java class named MainWindow. This class contains the Java code required to manage all the activities connected to this application. The main function is to load primary user interface with a connection to a
SQLite database. Every package, from group named java, has own module for specific build configuration named build.gradle. The third group creates a special folder to all noncode resources such as bitmap images, different strings and XML layouts and we recognize it with the name of res. When the app is started it runs in its own protected memory using a private virtual machine, so there is a high level of isolation against other apps. According to this behavior, this software project has a link only to those components that supplies all data requested by the app.

An entity-relationship model (ER) is required to understand connections between entities types located in the database. This ER is presented in Figure 3. 


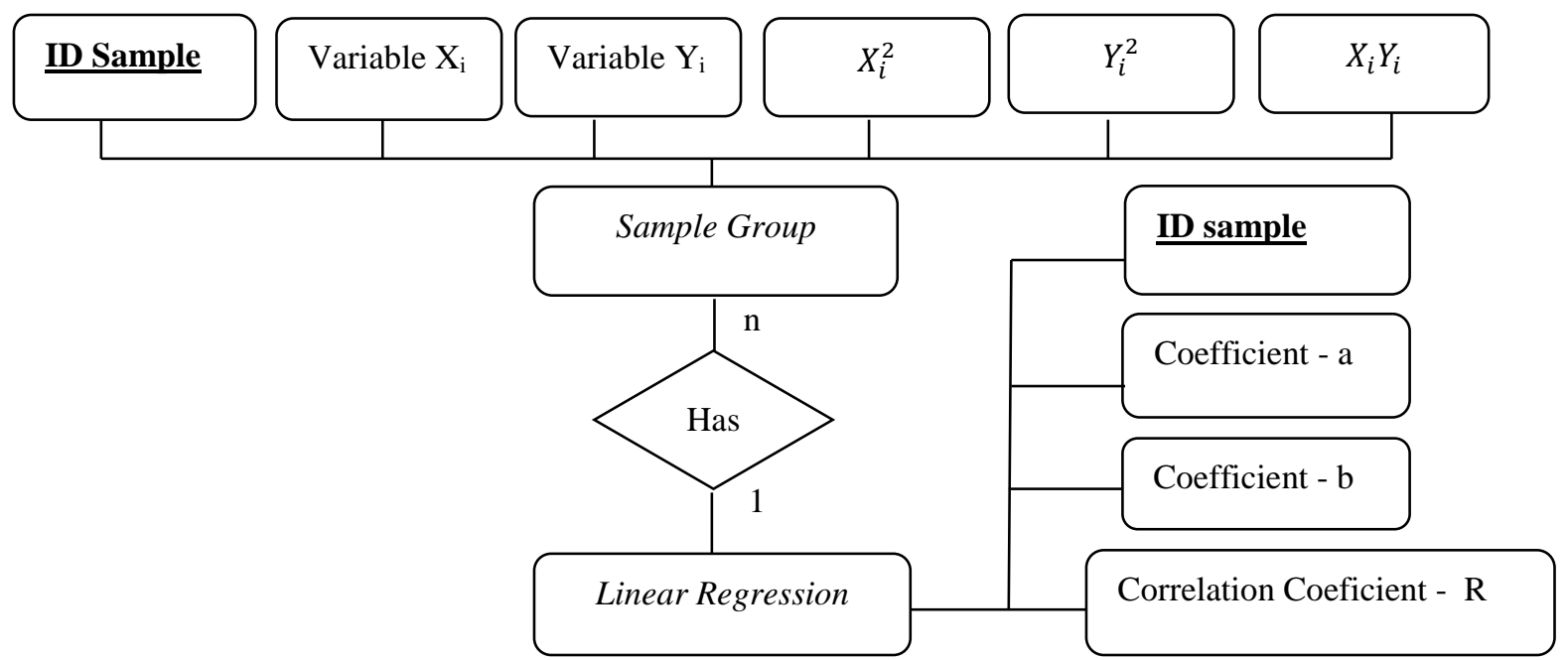

Fig. 3. Entity - relationship model for database implemented in application

From Figure 3 we observe that every sample group owns a group of $n$ variables $\left(\mathrm{Y}_{\mathrm{i}}, \mathrm{X}_{\mathrm{i}}\right)$ and we could say that the cardinalities of sample group and linear regression are $n$ and 1 respectively. There is a default solution in Android Studio to explore relationships between entities if we install plug-in named Code Iris. With this plug-in we obtain the following image presented in Figure 4. The black arrows from figure 4 are a reference to the fact that a new instance of DatabaseProject class is created at the level of the method onCreate for each class that extends AppCompatActivity. There is no support from this tool to find a graphical scheme correlated with the chain between activities inside application.

\section{C}

\section{DatabaseProject}

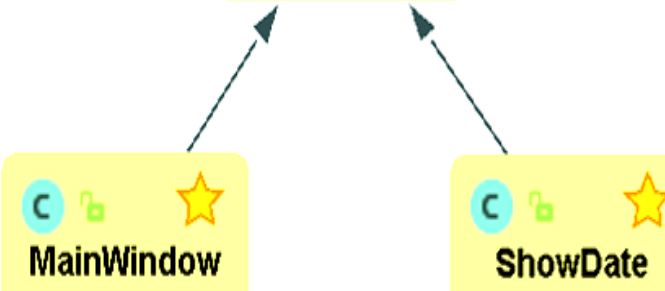

Fig. 4. Class view image with UML support provided by Code Iris

The class DatabaseProject creates the tables according to entity-relationship model. This class extends the class SQLiteOpenHelper because we are using SQLite as a default local database.The relationship between the table Reglin and Regcomp is the primary key named
Id and this key is shown in Figure 3 inside rectangle named ID Sample. Returning to MainWindow class, depicted in Figure 2, we envision a layout where user chooses an option according to his desire. 

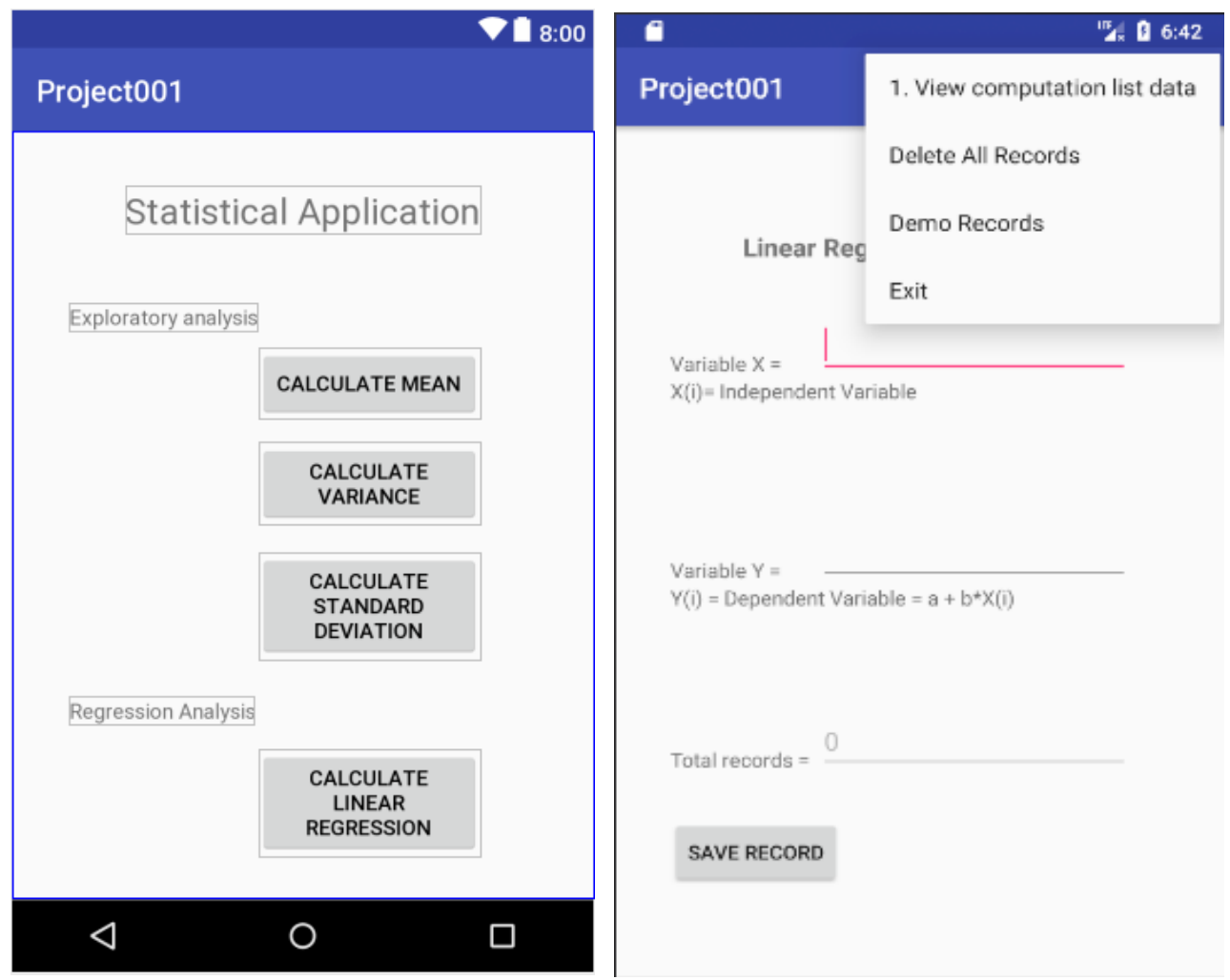

Fig. 5. The layout for the main window and the window for inserting pairs of values $\left(Y_{i}, X_{i}\right)$

Figure 5 presents the main window for the mobile application where user chooses an option to see how to solve theoretical aspects of the subject. There is a preview, in the Figure 5 at the right side, about one stage required when we speak about building the user interface such as when we create layout design with Android tools. The user interface selected to support inserting pairs of values $\left(\mathrm{Y}_{\mathrm{i}}, \mathrm{X}_{\mathrm{i}}\right)$ is presented in Figure 5, right. In order to store pairs of values $\left(\mathrm{Y}_{\mathrm{i}}, \mathrm{X}_{\mathrm{i}}\right)$ in tables of rows and columns we select SQLite database engine. This SQLite database solution offers the following facilities: no server backend, transactional and self-contained structure meaning that no other components are required. The class used to manage database is named SQLiteDatabase and all interactions starts from an instance of SQLiteOpenHelper that handles basic operations with the database. After a successfully saving of a pair of values $\left(\mathrm{Y}_{\mathrm{i}}, \mathrm{X}_{\mathrm{i}}\right)$ the blinking arrow of cursor restore its first position to enter a new data allocated to variable named $\mathrm{X}$. Meanwhile, the previous content for variable $\mathrm{Y}$ was emptied and the value of textbox allocated to total number of records was increased with one unit. If the user chooses from the main menu the first option then a new layout is activated. This new layout shows all calculations regarding the coefficient named $a, b$ and the Pearson's correlation coefficient $(R)$, as presented in Figure 6, at the left side. 


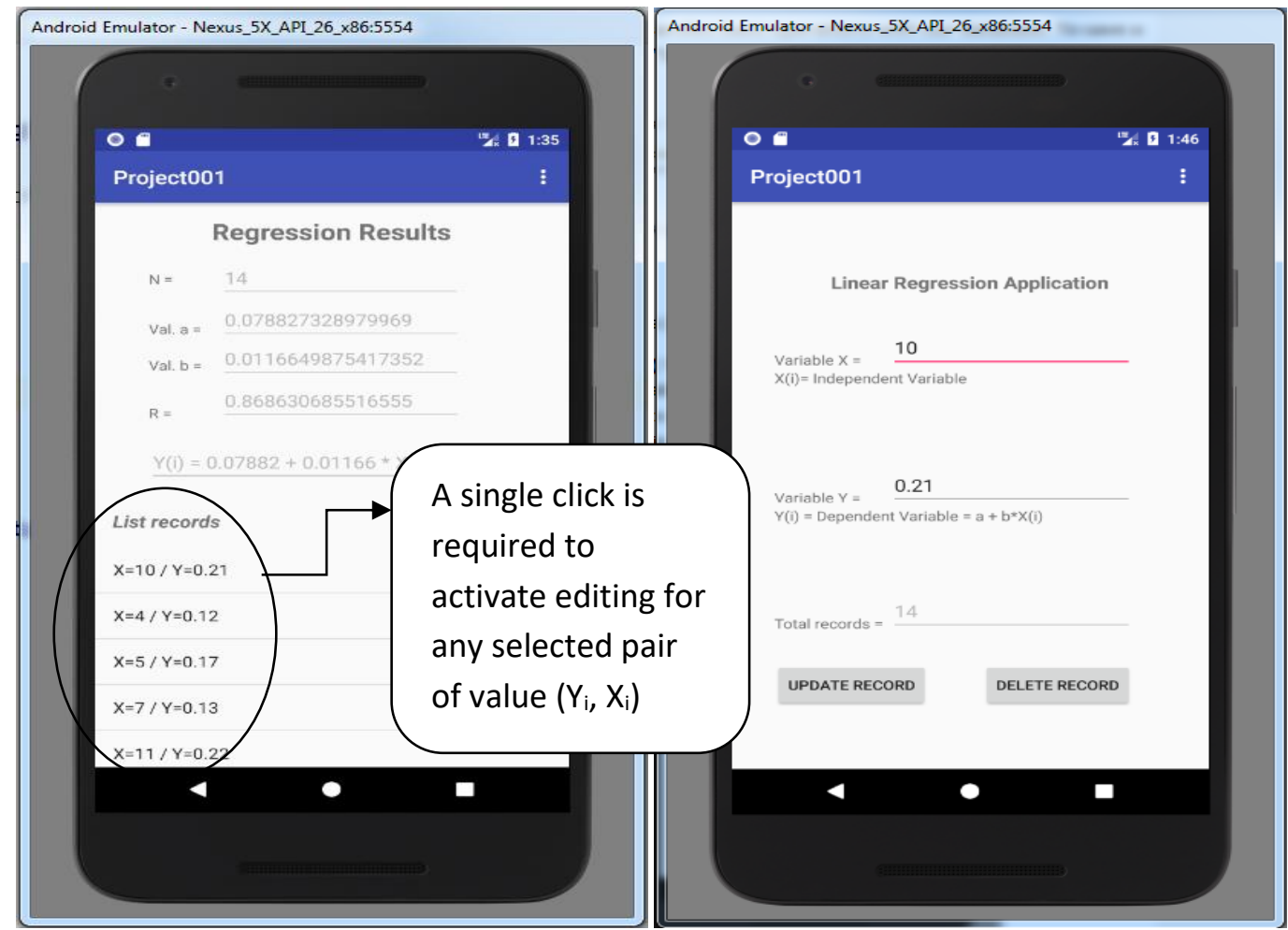

Fig. 6. A list of results including coefficient "a", "b" and correlation coefficient R - Pearson

If the user wants to modify or delete a pair of value $\left(\mathrm{Y}_{\mathrm{i}}, \mathrm{X}_{\mathrm{i}}\right)$ then a single click over a selected item from the list - object shown in figure 6 left is required. After this event the previous layout shown in figure 7 left will be restored with minor adjustments. The button named Save record will be renamed with Update record and a new button called Delete record will be activated. In this way the whole range of operations, insert - update - delete, were implemented in this Android project. The option named Delete All Records, shown in figure 6 left, delete all records from the database allocated to this project and the textbox labeled with generic name Total records will be cleared and a default 0 - value will be displayed. The last option from the main menu, shown in figure $6 \mathrm{left}$, called Demo records delete all rows and insert automatically 14 pairs of records values $\left(\mathrm{Y}_{\mathrm{i}}\right.$, $\mathrm{X}_{\mathrm{i}) \text {. }}$

\section{Interpretation}

The students who teach with a hands-on training based on this Android project have the opportunity to learn how to develop an Android application at the same time with simulating an economic experience of a well- known statistical method like linear regression. This approach could be seen as a method to increase knowledge by one addition after another in a cumulative manner for both academic branch studied at a university as we mention before: Econometrics and Programming Languages for Mobile Devices. Every student could be involved into a small or large project to develop a new project starting from a previous hands-on experience emulated during the classroom hours.

For our research, we consider there are at least two approaches for cross-disciplinary learning:

- The students will apply some concepts on another domain (like developing a mobile application used for econometrics);

- The students will use an application that helps them to learn some concepts by applying their current knowledge (like learning econometrics using their basic field of knowledge).

In this case study we will approach the first method: to develop an Android application for a generic econometric purpose considering that interpretation of results is the most important objective. This also represents a starting point for the second approach: an m- 
learning application.

Figure 7 presents the components involved in this kind of cross-disciplinary learning: inputs, processing and outputs. The most important part of this architecture is given by the students that combine several fields in order to achieve the results. The inputs are represented by application specifications and econometrics knowledge. The result of this process is represented by a mobile learning application.

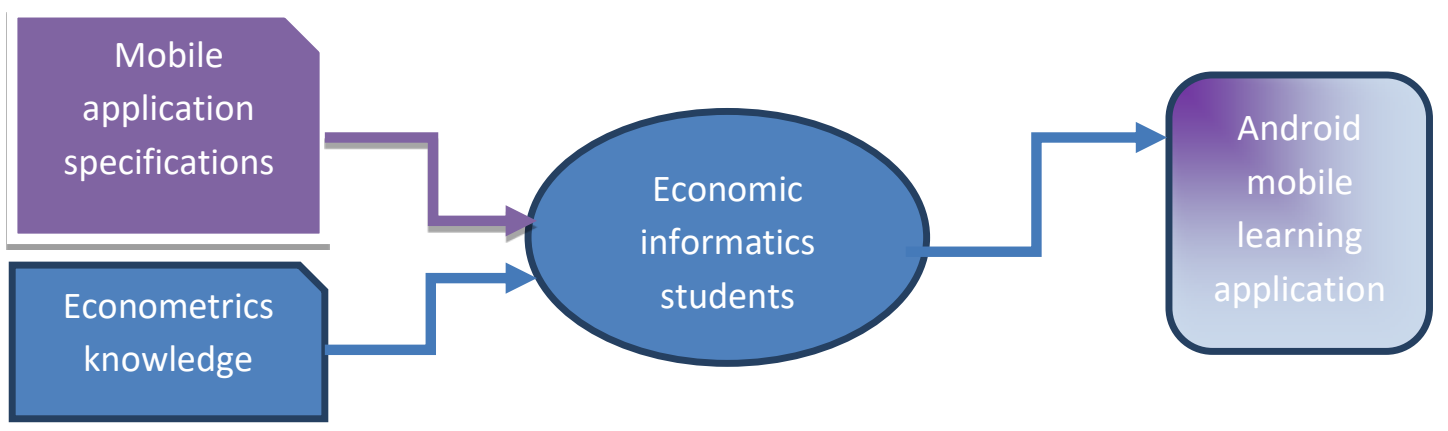

Fig. 7. Cross-disciplinary learning components

In order to achieve this objective (to learn different topics from other fields by using their current knowledge and skills), every input has to be well prepared. This requires a rigorous approach. Every process of development begins with a thought or a plan for a possible software project and could be assimilated with a picture in the mind. This stage involves market and science research. The starting idea progresses and a new state appears in which the main focus is located upon building the user interface.

The specifications of the mobile learning application should include UI guidelines based on researches on related to quality metrics based on user experience.

The results of this process have to be assessed. This requires measuring the initial knowledge related to the econometrics field and the knowledge after the application was developed. Also, the students will use the mobile learning application in order to improve their level of knowledge. We define the progress indicator $\left(P_{i}\right)$ to measure the progress made by the students at a moment of time $(i)$ :

$$
P_{i}=\frac{F k_{i}-I k}{I k}
$$

In (5), $F k_{i}$ represents the assessment result after the classes, and $I k$ represents the result of the initial assessment. $F k$ can be improved in time by using the developed application. The values of $P_{i}$ range from 0 to 1 . This is a relative indicator that has to be correlated with other indicators used for students' assessment. The proposed mobile learning solution is developed for Android platform. At this stage, the application's specifications are refined by following and testing the current steps and by developing UI related metrics. Several metrics presented in [19], such as mode of interaction (MI), drivability (D) or command speed (CS), will be used, as a starting point, to measure the application quality level.

The students who teach with a hands-on training based on this Android project have the opportunity to learn how to develop an Android application at the same time with simulating an economic experience of a wellknown statistical method like linear regression. This approach could be seen as a method to increase knowledge by one addition after another in a cumulative manner for both academic branch studied at a university as we mention before: econometrics and mobile applications development. Every student could be involved into a small or large project to develop a new project starting from a previous hands-on experience emulated during the classroom hours. 


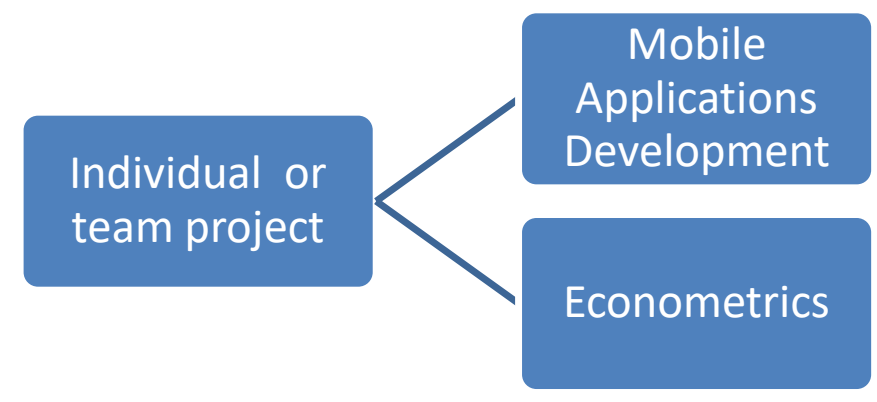

Fig. 8. A cross-disciplinary link between educational activities

We consider that knowledge accumulated during the course dedicated to learn about programming languages using mobile devices could be a better choice to manage an individual project targeting other courses like those where statistical methods are studied, instead using common solutions known as spreadsheet software. From a reverse point of view, the programming languages of mobile devices correlated with a rich experience gained at any course based on statistical methods will be a real advantage to any student and teacher (Figure 8).

\section{Conclusions and future work}

The final scope of this paper was attained by developing an m-learning Android application in order to ensure a better education according with the latest research caused by the useful utility of mobile devices in learning activities as we mention it. Teaching should have a triggering role for a better understanding, if students explain theory or how to solve practical problems to each other, not only for who is receiving knowledge but, also, to the student who is playing the role of a teacher. We think that learners need to be highly motivated to ensure a genuine attention during courses.

The following objectives will be considered to improve this current work:

- developing an application that assures more functionalities;

- generating automatically more default samples;

- searching from m-learning community a general view about those activities that really produce improvements for organizational model.

A great challenge for future directions about this work lies in developing a complex logical model to study m-learning capability regarding other exploring statistical methods like sample variance and standard deviation. Using cross-disciplinary learning approach allows teachers to generate immediate value and to manage a framework for collaboration. Another research direction will be the study the second approach for cross-disciplinary learning: to improve the m-learning application in order to help students to learn some concepts by applying their current knowledge. This requires identifying several metrics and characteristics related to these applications.

\section{Acknowledgment}

Parts of this research have been published in the Proceedings of the $17^{\text {th }}$ International Conference on Informatics in Economy, IE 2018 [20].

\section{References}

[1] T. Zahra, A. Amineh and M. Maryam, "The effect of m-learning on mathematics learning," Procedia - Social and Behavioral Sciences, vol. 171, pp. 83-89, 2015.

[2] W. Zoraini, L. Chng and M. Norziati, "A study on learner readiness for mobile learning at Open University Malaysia," in IADIS International Conference Mobile Learning, 2009.

[3] M.-T. Barbaux, "From lifelong learning to m-learning.," in 13th International Conference Association for Learning Technology, The next generation., HeriotWatt University Edinburgh, Scotland, UK, 2006. 
[4] A. Kieth and J.-R. Winquist, "Evaluating and active learning approach to teaching introductory statistics : A classroom workbook approach," Journal of Statistics Education, vol. 19, no. 1, 2011.

[5] T. J. Pfaff and A. Weinberg, "Do handson activities increase student understanding?: A case study," Journal of Statistics Education, vol. 17, no. 3, 2009.

[6] S. Knypstra, "Teaching Statistics in an Activity Encouraging Format," Journal of Statistics Education, vol. 17, no. 2, 2009.

[7] J.-B. Bottentuit, D. Negretti and P. Coutinho, "Methodology to use Multimedia Applications and Mobile Devices When Teaching Structural Analysis," in International Conference on Engineering Education - ICEE, Coimbra, Portugal, September 3-7, 2007.

[8] M. Tilca, "Mobile computing using Android with an emphasizes on economic application," Network Intelligence Studies, vol. IV, no. 2(8), 2016.

[9] A.-M. Ion, C.-G. Apostol and D. Vespan, "Optimizarea interfetelor platformelor educationale," in RoCHI, 2012.

[10] B. Samsiah, "Adoption and application of mobile learning in the education industry," Procedia - Social and Behavioral Sciences, vol. 90, pp. 720-729, 2013.

[11] A. Iancu and D.-M. Ionescu, "Learning Analytics Mobile Application for the Bucharest University of Economic Studies' Students," Informatica Economica, vol. 21, no. 3, pp. 65-72, 2017.

[12] C.-S. Ding, "Using regression mixture analysis in educational research," Practical Assessment Research \& Evaluation, vol. 11, no. 11, December 2006.
[13] J. Butviliene and T. Butvilas, "The Regression Model For Adults Participation In Non-Formal Education: Public vs. Private Sectors.," Innovative Infotechnologies for Science, Business and Education, vol. 1, no. 14, pp. 10-14, 2013.

[14] R. Margea and C. Margea, "Considerations for the Mobile Web. Paradigm Shift," Informatica Economica, vol. 21, no. 1, pp. 16-29, 2017.

[15] I. Goksu and B. Atici, "Need For Mobile Learning: Technologies and Opportunities," Procedia - Social and Behavioral Sciences, vol. 103, pp. 685694, 2013.

[16] M. Rahimi and S. Miri, "The Impact of Mobile Dictionary Use on Language Learning," Procedia - Social and Behavioral Sciences, vol. 98, pp. 14691474, 2014.

[17] D.-S. Hamdani, "Mobile Learning: A Good Practice," Procedia - Social and Behavioral Sciences, vol. 103, pp. 665674, 2013.

[18] D. Fowler, "List of Alternative Android App Development IDEs," 2017. [Online]. Available: https://tekeye.uk/android/listof-android-app-development-ides.

[Accessed 7 March 2018].

[19] P. Pocatilu, I. Ivan, A. Zamfiroiu and C. Boja, "An aggregate indicator for mobile application quality assessment," Turkish Journal of Electrical Engineering \& Computer Sciences, vol. 23, no. 4, pp. 956-973, 2015.

[20] D. Mihail-Vaduva, A Mobile Solution for Cross-Disciplinary Learning, Proc. of. the 17th International Conference on Informatics in Economy (IE 2018), pp. 119-124, 17-20 May 2018, ISSN 22847472 
Dinu MIHAIL-VĂDUVA has graduated the Faculty of Economic Cybernetics, Statistics and Informatics of the Bucharest Academy of Economic Studies in 2010. He received an alumnus achievement award in 2012 of the Informatics Economics Master affiliated to the Academy of Economic Studies from Bucharest. Starting with 2017 he was admitted to the $\mathrm{PhD}$ student at the Doctoral School of The Bucharest University of Economic Studies, Economic Informatics domain for his $\mathrm{PhD}$ thesis proposal involving educational systems based on mobile technologies coordinated by Professor dr. Paul POCATILU. Between 2016 and 2017 he finished with a high school diploma postgraduate training programs named Psychological, Pedagogical and Educational skills training, level I and II, connected with a particular branch of the Bucharest University of Economic Studies. Furthermore, he graduated classes between 1984 and 1989 with a bachelor degree of the Faculty of Technology for Chemistry of the Polytechnic Institute of Bucharest towards specialization concerning Inorganic Chemistry Engineering. Currently he is working as a software analyst within the Department of Information Technology at the Regia Autonomă "Monitorul Oficial" from Bucharest and he is using Oracle database with APEX technology correlated with specific software languages for intranet projects development. His main scientific preoccupation is heavily orientated towards domains situated at the interface between education as a primarily economic and social activity and the most recent software technology. 